\title{
Pancreatic schwannoma: Report of a case and review of literature
}

\author{
Vidyadhar A. Kinhal · Ravishankar T. H. S. • Ashok I. Melapure • G. Jayaprakasha • Range Gowda B. C. • Manjunath
}

Received: 6 December 2007 / Accepted: 10 January 2010

(C) Association of Surgeons of India 2010

\begin{abstract}
Connective tissue tumors of pancreas are uncommon, among them pancreatic schwannoma is very rare tumor, very few cases were reported in literature. Aggressive resections like whipple's procedure, or distal pancreatectomy are not necessary for pancreatic schwannoma as it rarely goes to malignant change and simple enuclation is sufficient.

In our patient pancreatic schwannoma is associated with simple cyst in liver and absence of one kidney. Here, we are presenting a case of pancreatic schwannoma treated by simple enucleation.
\end{abstract}

Keywords Pancreatic schwannoma $\cdot$ Simple cyst liver, absence of kidney $\cdot$ Simple enucleation

V. A. Kinhal · Ravishankar T. H. S. · A. I. Melapure ·

G. Jayaprakasha $\cdot$ R. Gowda B. C. · Manjunath

Department of General Surgery,

Vijayanagar Institute of Medical Sciences (VIMS),

Bellary - 583104, Karnataka, India

Ravishankar T. H. S. ( $\bowtie)$

E-mail: raviths@rediffmail.com

\section{Introduction}

Schwannomas are rare neoplasms. Commonly originate in 8th cranial nerve although other cranial or peripheral nerves are affected [1]. Pancreatic schwannomas are even more unusual neoplasms. Only 26 patients being reported in the english literature from medline search. $2 / 3$ of these tumors are reported to undergo cystic degeneration and can cause clinical confusion with pancreatic pseudocyst. In this respect we are sharing our experience with a case of pancreatic schwannoma, with an emphasis that simple enucleation is sufficient. Our patient also had associated absence of one kidney and simple cyst in liver, which is not reported earlier.

\section{Case report}

A 55-year-old man presented with vague upper abdominal pain of 4 months. There was no other remarkable history. On examination of his abdomen an illdefined mass felt in epigastric region with mild hepatomegaly. Preoperative work up of this patient was quite interesting. All haematological investigations were within normal limits, including haemogram, liver and renal function tests and serum amylase levels. Ultrasound abdomen showed a complex echogenic retroperitoneal mass of $53 \times 43 \times 68 \mathrm{~mm}$ with cystic area caudal to tail of pancreas, non-visualization of right kidney, with hypertrophy of left kidney and a simple cyst in liver (Fig. 1). Intravenous urogram showed non-excretion of contrast on right side (Fig. 1). Computed tomography (CT) scan, magnetic resonance imaging (MRI) of the abdomen were not done as these facilities were not available in the institution. Ultrasonography (USG) guided fine needle aspiration cytology was inconclusive. Patient 


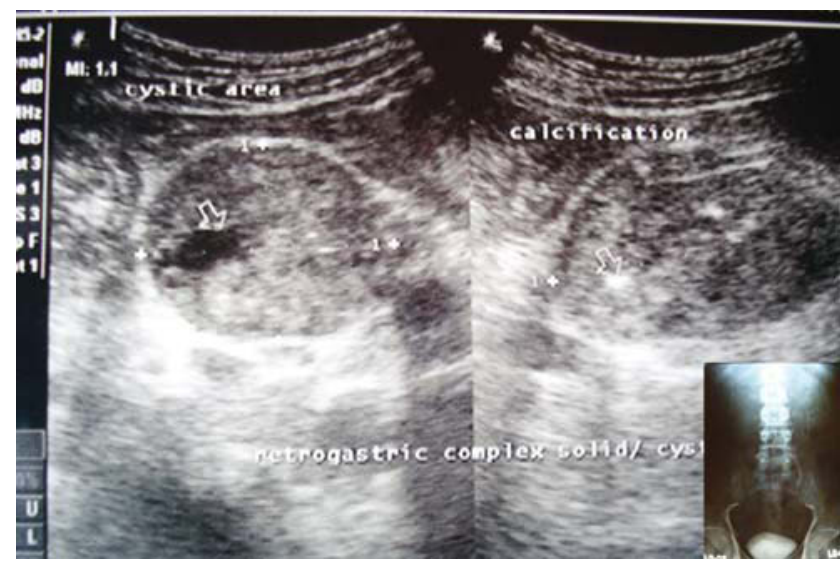

Fig. 1 Ultrasonography showing solid-cystic mass in pancreas. Inset showing intravenous urography with non excretion of contrast on right side

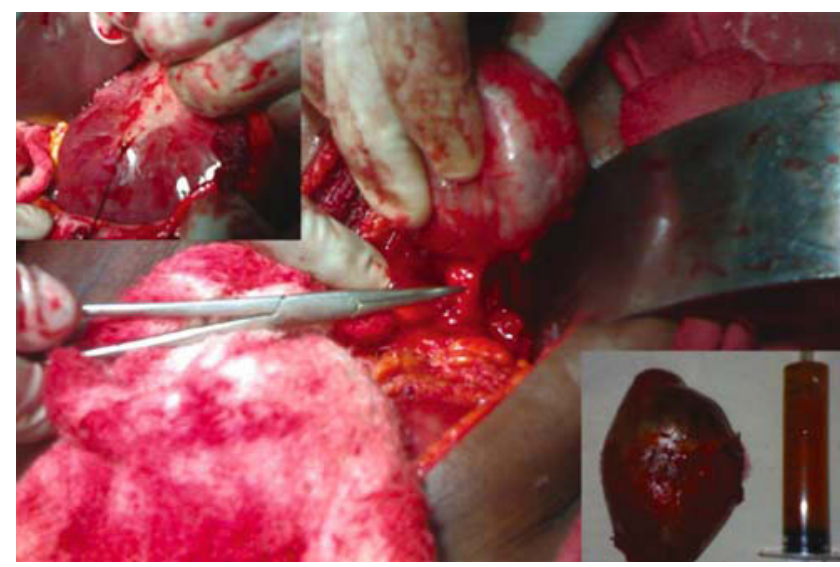

Fig. 2 Operative photo showing pancreatic schwannoma with flimsy adhesions to superior mesenteric artery and being enucleated. Top inset: simple cyst of liver. Bottom inset: enucleated specimen

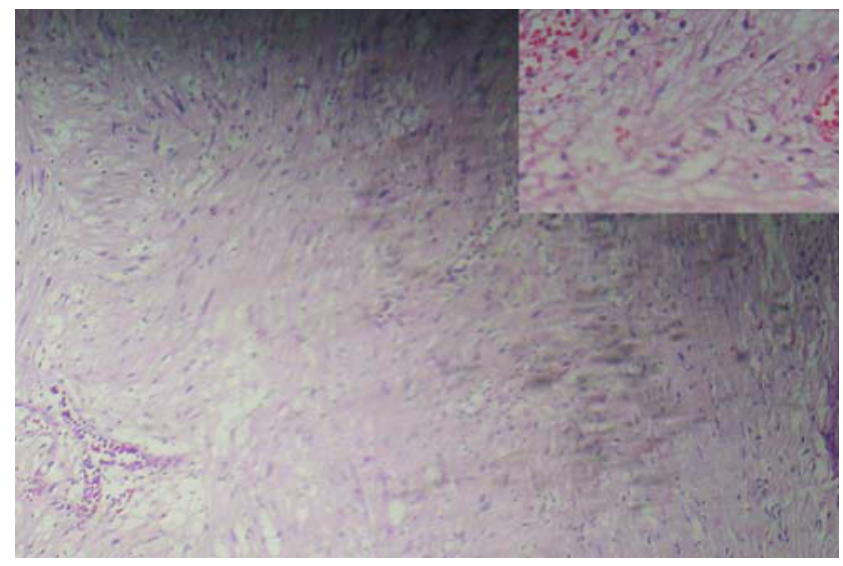

Fig. 3 Histological section showing cellular (Antoni A) area with spindle cells having wavy, undulating nuclei arranged in pallisading manner and hypocellular (Antoni B) areas. Inset: high-power field showing blood vessel and spindle cells with no features of malignancy underwent exploratory laparotomy. A simple cyst in right lobe of liver found which was deroofed and drained. Right kidney was absent with compensatory hypertrophy of left kidney. A cystic-solid mass was seen arising mainly from the body of pancreas extending in to the head. The tumor was well encapsulated with few flimsy adhesions to superior mesenteric artery (Fig. 2). The tumor was not fixed to adjacent structures. There was no evidence of any intraperitoneal metastases. Enucleation of the tumor performed. Drainage placement preceded abdominal closure. The patient recovered uneventfully and was discharged on 10th hospital day.

Pathological examination of the specimen reported as schwannoma (Fig. 3). No post operative chemotherapy or radiotherapy scheduled. He was followed for a period of three months during which he was relieved of his symptoms with no evidence of recurrence.

\section{Discussion}

Tumors of connective tissue origin are very rare in pancreas. But a wide spectrum has been reported like fibrosarcoma, leiomyosarcoma, haemangiopericytoma, histiocystoma, lymphoma and schwannoma [2, 3]. Only 26 cases of pancreatic schwannoma have been reported in english literature mainly as case reports, as found using a medline search.

Benign schwannomas are derived from schwann cells, the lining cells of nerve sheath. Common sites of origin include head and neck region, the extremities and the trunk. Rarely schwannoma can involve stomach, liver, small and large bowel, lesser sac, fallopian tubes and pancreas [4]. Pancreatic schwannoma arise from either autonomic sympathetic or para sympathetic fibres, both of which course through pancreas via vagus [4]. Benign schwannomas grow slowly and these tumors vary considerably in size and approximately two thirds are partially cystic, which can cause clinical confusion as pseudocyst of pancreas [5]. Cystic schwannoma is a rare neoplasm occurring in the pancreas and should be considered in the differential diagnosis for patients presenting with pancreatic cystic lesions [6, 7].

Commonly seen in adults with almost equal male to female ratio. Non-specific abdominal pain is the most commonly reported symptom. Majority of these tumors located in the head (42.3\%) and body $(23 \%)$ of the pancreas [8]. Majority of tumors were benign $(80.76 \%)$, but there were five reported malignancies (19.23\%) [9]. Reported cases of intrapancreatic schwannoma have recently increased in the literature. However, none of these cases were diagnosed as schwannoma preoperatively [10]. Preoperative diagnosis of schwannoma is difficult. CT scan is often useful to establish pancreatic lesion. CT findings in pancreatic schwannoma however are similar to non-pancreatic schwannoma and 
usually demonstrate well defined and hypodense tumors with encapsulation and cystic degeneration [4, 8]. MRI is helpful in characterizing their typical encapsulation, hypointensity on $\mathrm{T} 1$ - weighted images and hyperintensity on T2. It may also reveal vascular involvement which can further characterize the lesion as malignant $[4,11]$. Our patient with pancreatic schwannoma was associated with simple cyst in liver and absence of right kidney. In review of literature no such association was found in any of the case reports. Review of literature shows operable pancreatic schwannomas were treated by whipple resection, distal pancreatectomy and simple enucleation. Since malignant transformation is uncommon, simple enucleation is feasible, safe and sufficient. Early detection and early treatment of tumor were helpful in increasing the ratio of enucleation and avoiding a very extensive pancreatic resection $[8,12]$.

To conclude, although pancreatic schwannoma is rare, it should be considered for differential diagnosis of pancreatic cystic lesions and simple enucleation of the tumor is recommended.

\section{References}

1. Vincet T Devita, Jr Samuel Helman, Steven A Rosenberg CANCER: Principle and Practice of Oncology 4th edition: Vol 2

2. Carlos Fernandez-Del, Castillo, Andrew L Warshaw Pancreatic cancer: Oxford text book of surgery: Volume 1 section 25:1309-1317

3. Russell RCG The Pancreas. Bailey and love's short practice of surgery. 24th edition: 1114-1132

4. Bruce A Urban, Elliot K Fishman, Ralph H Hruban, John L Cameron (1992) CT findings in cystic schwannoma of the pancreas. J Computer Assisted Tomography 16(3):492-496

5. Brown SZ, Owen DA, O'connell JX, Scudamore CH (1998) Schwannoma of the pancreas: A report of two cases and a review of the literature. Mod Pathol 11(12):1178-1182

6. Kristi M Almo MD, L William Traverso MD (2001) Pancreatic schwannoma: An uncommon but important entity. J Gastrointestinal Surgery 5:359-363

7. Ital J (1993) The schwannoma: An uncommon type of cystic lesion of the pancreas. Gastroenterol 25(7):385-387

8. Bui TD, Nguyen T, Huertas, Gu M, Hsiang D (2004) Pancreatic Schwannoma. A case report and review of the literature: JOP. 10;5(6):520-526

9. Paranjape C, Johnson SR, Khwaja K, Goldman H, Kruskal JB, Hanto DW (2004) Clinical characteristics, treatment, and outcome of pancreatic schwannomas. J Gastrointest Surg 8:706-712

10. Soumaoro LT, Teramoto K, Kawamura T, Nakamura N, SanadaT, Suginara K, Ariis. Benign Schwannoma of the Pancreas J Gastrointest Surg Feb;9(2):288-290

11. Novellas S, Chevallier P, Saint Paul MC, Gugenheim J, Bruneton JN (2005) MRI Features of a pancreatic schwannoma clin imaging 29(6):434-436

12. Wu YL, Yan HC, Chen LR, Chen J, Gao SL, Li JT (2005) Pancreatic benign schwannoma treated by simple enucleation: case report and review of literature. Pancreas 31(3):286-288 